\title{
DIRECT ONE-STEP METHOD FOR SOLVING THIRD-ORDER BOUNDARY VALUE PROBLEMS
}

\author{
Athraa Abdulsalam ${ }^{1,3}$, Norazak Senu ${ }^{1,2}$, Zanariah Abdul Majid ${ }^{1,2}$ \\ ${ }^{1}$ Department of Mathematics \\ Universiti Putra Malaysia \\ 43400 UPM Serdang, Selangor, MALAYSIA \\ ${ }^{2}$ Institute for Mathematical Research \\ Universiti Putra Malaysia \\ 43400 UPM Serdang, Selangor, MALAYSIA \\ ${ }^{3}$ Department of Mathematics \\ University of Baghdad \\ Al-Jadrriya, Baghdad, IRAQ
}

\begin{abstract}
A direct explicit Runge-Kutta type (RKT) method via shooting technique to approximate analytical solutions to the third-order two-point boundary value problems (BVPs) with boundary condition type I and II are proposed. In this paper first, a three-stage fourth-order direct explicit RungeKutta type method denoted as RKT3s4 is constructed. A new algorithm of shooting technique for solving two-point BVPs for third-order ordinary differential equations (ODEs) is presented.
\end{abstract}

AMS Subject Classification: 65L05, 65L06, 65L20

Key Words: direct Runge-Kutta method, two-point boundary value problem, shooting technique, third-order ordinary differential equation

Received: August 16, 2018

(c) 2019 Academic Publications

${ }^{\S}$ Correspondence author 


\section{Introduction}

Numerous problems in mathematics can be formulated in the form of differential equations, an initial value problem (IVP) is an ordinary differential equation (ODE) whose boundary conditions are specified at a single point, which can be found in mathematical modeling of real-life problems [1]-[3]. There is also another class of the ODE which is the boundary value problem (BVP), a BVP differs from an IVP in that the boundary conditions are specified at more than one point and in that solutions of the differential equation over an interval, satisfying the boundary conditions at the endpoints, are required ([4], p.1). BVP arises in several branches of engineering and applied sciences including fluid dynamics and chemical reactions, elastic beams, etc. (see [5], p.7 - p.27).

In this work, we concentrate on finding an approximate solution to twopoint BVPs with boundary conditions type I and II of the form

$$
u^{\prime \prime \prime}=f(t, u), \quad a<t<b,
$$

with boundary conditions:

(a) Type I

$$
u(a)=\alpha, \quad u^{\prime}(a)=\beta, \quad u(b)=\gamma .
$$

(b) Type II

$$
u(a)=\alpha, \quad u^{\prime}(a)=\beta, \quad u^{\prime}(b)=\lambda .
$$

where $a, b, \alpha, \beta, \gamma$, and $\lambda$ are constants, the proof of existence and uniqueness of solutions to two-point BVPs of third-order ODE is possible, see Henrici [6]. For analytical solutions of the IVPs and BVPs, analytical methods are seldom used since most of the problems encountered were difficult, with either complex differential equations or complex boundary conditions ([7], [8]) or sometimes finding an analytical solution to some ODE applications is complicated or impossible, therefore recourse to numerical methods in such cases is almost the only choice. For several years, various numerical methods have been derived to handle the IVPs and BVPs, which is a subject can be treated separately. The numerical procedures for the solution of the IVP can be classified into two major groups: one-step methods and multi-step methods. One of the advantages of one-step methods is that it can change the step size easily at different $t$ ([4], p.10). Two such methods of the one-step are Taylor's method and Runge-Kutta (RK) method. The numerical algorithm of the RK method is considered the most widely used scheme, due to its low truncation error ([4], p.8). For the 
multi-step, such methods are Predictor-Corrector methods, Adams-Moulton method, Adams -Bashforth method, and Trapezium rule method. Similarly, for the numerical procedures for the solution of the BVP, there exist several techniques, such as Finite-Difference method ([9], [10]), Shooting method ([5], $[11])$, the quasilinearization method ([12], [13]), the monotone iterative method ([14], [15]), and the variational iteration method ([16], [17]). Burden and Faires [1] have used RK method (after transferring it into the system of first-order) via shooting technique to solve second-order two-point linear BVP with Dirichlet boundary condition. However, many researchers ([18], [19]) have shown that using direct RK approach to solve higher-order ODE without reducing it first to a system of first-order is superior and more efficient than the conventional RK. Where there is no need to increase the number of equations and calculating more function evaluations which lead to a time-consuming process and more human effort as in classical RK.

Therefore, the purpose of this study is to construct a direct and effective method of Runge-Kutta type with less computation time and function evaluations to solve two-point BVPs of third-order with boundary conditions type I and II, algorithm of shooting technique was offered to develop the approximate analytical solutions.

The organizing of this paper is as follows: In Section 2, the construction of the explicit RKT3s4 method is presented. The explanation of the new shooting technique algorithm is given in Section 3. In Section 4, four problems numerically examined the efficiency of the RKT3s4 method as compared to the existing method and the last section, deals with the conclusions.

\section{Construction of the Explicit RKT3s4 Method}

In this section, an explicit three-stage RKT method of order-four will be derived. The general $\mu$-stage RKT method for the differential equation

$$
u^{\prime \prime \prime}=f(t, u(t))
$$

given by

$$
\begin{aligned}
& u_{n+1}=u_{n}+h u_{n}^{\prime}+\frac{h^{2}}{2} u_{n}^{\prime \prime}+h^{3} \sum_{i=1}^{\mu} b_{i} \kappa_{i}, \\
& u_{n+1}^{\prime}=u_{n}^{\prime}+h u_{n}^{\prime \prime}+h^{2} \sum_{i=1}^{\mu} \hat{b}_{i} \kappa_{i},
\end{aligned}
$$




$$
\begin{array}{c|ccccc}
c_{1} & 0 & & & & \\
c_{2} & a_{21} & & & & \\
c_{3} & a_{31} & a_{32} & & & \\
\vdots & \vdots & \vdots & & & \\
c_{\mu} & a_{\mu, 1} & a_{\mu, 2} & \ldots & a_{\mu, \mu-1} & \\
& & & & & \\
\hline & b_{1} & b_{2} & \ldots & b_{\mu-1} & b_{\mu} \\
& \hat{b}_{1} & \hat{b}_{2} & \ldots & \hat{b}_{\mu-1} & \hat{b}_{\mu} \\
& \hat{\hat{b}}_{1} & \hat{\hat{b}}_{2} & \ldots & \hat{b}_{\mu-1} & \hat{b}_{\mu}
\end{array}
$$

Table 1: $\mu$-stage explicit RKT method.

$$
u_{n+1}^{\prime \prime}=u_{n}^{\prime \prime}+h \sum_{i=1}^{\mu} \hat{\hat{b}}_{i} \kappa_{i}
$$

where

$$
\begin{aligned}
& \kappa_{1}=f\left(t_{n}, u_{n}\right), \\
& \kappa_{i}=f\left(t_{n}+c_{i} h, u_{n}+c_{i} h u_{n}^{\prime}+\frac{h^{2}}{2} c_{i}^{2} u_{n}^{\prime \prime}+h^{3} \sum_{j=1}^{\mu} a_{i j} \kappa_{j}\right),
\end{aligned}
$$

for $i=2,3, \ldots, \mu$, where $c_{i}, a_{i j}, b_{i}, \hat{b}_{i}$, and $\hat{\hat{b}}_{i}$ for $i=1,2, \ldots, \mu$ and $j=$ $1,2, \ldots, \mu$ are the parameters of the RKT method and they supposed to be real. RKT method is said to be explicit when $a_{i j}=0$ for $i \leq j$, otherwise it is an implicit method. RKT method (5)-(8) can be written by the well-known Butcher tableau as follows (see Table 1):

To derive the RKT3s4 method, we will use the order conditions that have given by Mechee et al. [20] up to fifth-order for $u, u^{\prime}$, and $u^{\prime \prime}$. The order conditions of $\mu$-stage RKT method up to fifth-order as given in [20] are given as follows.

The order conditions for $u$ :

Order 3:

$$
\sum b_{i}=\frac{1}{6}
$$

Order 4:

$$
\sum b_{i} c_{i}=\frac{1}{24}
$$


Order 5:

$$
\sum b_{i} c_{i}^{2}=\frac{1}{60}
$$

The order conditions for $u^{\prime}$ :

Order 2:

$$
\sum \hat{b}_{i}=\frac{1}{2}
$$

Order 3:

$$
\sum \hat{b}_{i} c_{i}=\frac{1}{6}
$$

Order 4:

$$
\sum \hat{b}_{i} c_{i}^{2}=\frac{1}{12}
$$

Order 5:

$$
\sum \hat{b}_{i} c_{i}^{3}=\frac{1}{20}, \quad \sum \hat{b}_{i} a_{i j}=\frac{1}{120} .
$$

The order conditions for $u^{\prime \prime}$ :

Order 1:

$$
\sum \hat{\hat{b}}_{i}=1
$$

Order 2:

$$
\sum \hat{\hat{b}}_{i} c_{i}=\frac{1}{2}
$$

Order 3:

$$
\sum \hat{\hat{b}}_{i} c_{i}^{2}=\frac{1}{3}
$$

Order 4:

$$
\sum \hat{\hat{b}}_{i} c_{i}^{3}=\frac{1}{4}, \quad \sum \hat{\hat{b}}_{i} a_{i j}=\frac{1}{24},
$$

Order 5:

$$
\sum \hat{\hat{b}}_{i} c_{i}^{4}=\frac{1}{5}, \quad \sum \hat{\hat{b}}_{i} a_{i j} c_{j}=\frac{1}{120}, \quad \sum \hat{\hat{b}}_{i} a_{i j} c_{i}=\frac{1}{30} .
$$

Now, assume that $c_{3}=1$, as a result, we will have a system of nonlinear equations, consisting of ten nonlinear equations with fourteen unknown variables that have not yet been resolved, as follows:

$$
\begin{aligned}
& b_{1}+b_{2}+b_{3}=\frac{1}{6}, \\
& b_{2} c_{2}+b_{3} c_{3}=\frac{1}{24}, \\
& \hat{b}_{1}+\hat{b}_{2}+\hat{b}_{3}=\frac{1}{2},
\end{aligned}
$$




$$
\begin{aligned}
& \hat{b}_{2} c_{2}+\hat{b}_{3} c_{3}=\frac{1}{6}, \\
& \hat{b}_{2} c_{2}^{2}+\hat{b}_{3} c_{3}^{2}=\frac{1}{12}, \\
& \hat{\hat{b}}_{1}+\hat{\hat{b}}_{2}+\hat{\hat{b}}_{3}=1, \\
& \hat{\hat{b}}_{2} c_{2}+\hat{\hat{b}}_{3} c_{3}=\frac{1}{2}, \\
& \hat{\hat{b}}_{2} c_{2}^{2}+\hat{\hat{b}}_{3} c_{3}^{2}=\frac{1}{3}, \\
& \hat{\hat{b}}_{2} c_{2}^{3}+\hat{\hat{b}}_{3} c_{3}^{3}=\frac{1}{4}, \\
& \hat{\hat{b}}_{2} a_{2,1}+\hat{\hat{b}}_{3} a_{3,1}+\hat{\hat{b}}_{3} a_{3,2}=\frac{1}{24} .
\end{aligned}
$$

Accordingly, the system has a solution based on three free parameters $b_{2}$, $a_{2,1}$, and $a_{3,1}$ as follows:

$$
\begin{aligned}
\hat{\hat{b}}_{1} & =\frac{1}{6}, \\
\hat{\hat{b}}_{2} & =\frac{2}{3} \\
\hat{\hat{b}}_{3} & =\frac{1}{6} \\
a_{3,2} & =-4 a_{2,1}-a_{3,1}+\frac{1}{4}, \\
b_{1}= & \frac{1}{8}-\frac{1}{2} b_{2}, \\
b_{3} & =-\frac{1}{2} b_{2}+\frac{1}{24}, \\
c_{2} & =\frac{1}{2}, \\
c_{3} & =1, \\
\hat{b}_{1} & =\frac{1}{6}, \\
\hat{b}_{2} & =\frac{1}{3}, \\
\hat{b}_{3} & =0 .
\end{aligned}
$$

Based on Dormand [21], the free parameters are chosen by minimizing the error equations. The global error of the fifth-order conditions is defined as 


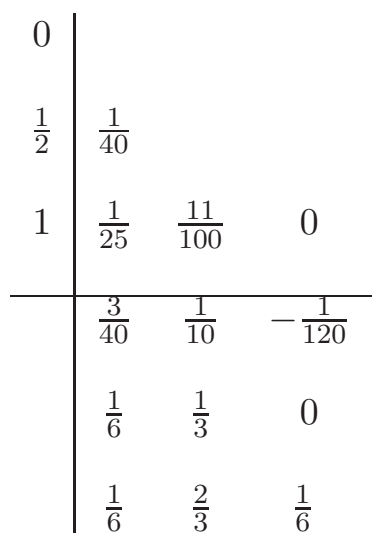

Table 2: Butcher tableau for RKT3s4 method.

follows:

$$
\left\|T_{g}^{(5)}\right\|_{2}=\sqrt{\sum_{i=1}^{n_{p+1}^{\prime \prime}}\left(T_{i}^{\prime \prime(5)}\right)^{2}+\sum_{i=1}^{n_{p+1}^{\prime}}\left(T_{i}^{\prime(5)}\right)^{2}+\sum_{i=1}^{n_{p+1}}\left(T_{i}^{(5)}\right)^{2}}
$$

where $T^{\prime \prime(5)}, T^{\prime(5)}$, and $T^{(5)}$ are the local truncation error terms of the RKT methods for $u^{\prime \prime}, u^{\prime}$, and $u$ respectively and $T_{g}^{(5)}$ is the global truncation error. Based on the free parameters $b_{2}, a_{2,1}$, and $a_{3,1}$ we get the global truncation error term of fifth-order condition for $u, u^{\prime}, u^{\prime \prime}$ as follows:

$$
\begin{aligned}
\left\|T_{g}^{(5)}\right\|_{2}= & \frac{1}{240}\left(19200 a_{2,1}^{2}+3200 a_{2,1} a_{3,1}+400 a_{3,1}^{2}+3600 b_{2}^{2}\right. \\
& \left.-1120 a_{2,1}-120 a_{3,1}-720 b_{2}+61\right)^{1 / 2} .
\end{aligned}
$$

Then, minimizing equation (43) with respect to the free parameters $b_{2}, a_{2,1}$, and $a_{3,1}$ by using Maple software (Minimize command in Optimization package) to obtain $b_{2}=\frac{1}{10}, a_{2,1}=\frac{25}{1000}, a_{3,1}=\frac{4}{100}$, and $\left\|T_{g}^{(5)}\right\|_{2}=0.01181453907$. Lastly, all the coefficients of the RKT3s4 method are written in Butcher tableau (see Table 2):

\section{Shooting Method for Linear BVP}

Shooting technique is used to convert the BVP to IVPs. The idea of shooting technique is to obtain the missing initial value until the boundary condition at 
the other end converges to its correct value. When we use the shooting method, we transform (1) into IVP of the form

$$
\begin{aligned}
u^{\prime \prime \prime} & =f(t, u), \quad a<t<b, \\
u(a) & =\alpha, \quad u^{\prime}(a)=\beta, \quad u^{\prime \prime}(a)=\lambda_{1},
\end{aligned}
$$

where $\lambda_{1}$ is any number. Then the resulting IVP will be solved using RKT method.

\section{Reduction to Three IVPs:}

The solution of a linear two-point BVP is associated with the formation of a linear combination of the solutions to three IVPs.

The form of the IVPs as follows.

Suppose that $\psi(t)$ is the unique solution to the IVP

$$
\begin{aligned}
\psi^{\prime \prime \prime} & =f_{1}(t, \psi), \\
f_{1}(t, \psi) & =q(t) \psi(t)+g(t), \text { with } \psi(a)=\alpha, \psi^{\prime}(a)=0, \psi^{\prime \prime}(a)=0 .
\end{aligned}
$$

Suppose that $\rho(t)$ is the unique solution to the IVP

$$
\begin{aligned}
\rho^{\prime \prime \prime} & =f_{2}(t, \rho), \\
f_{2}(t, \rho) & =q(t) \rho(t), \text { with } \rho(a)=0, d \rho^{\prime}(a)=1, \rho^{\prime \prime}(a)=0 .
\end{aligned}
$$

Suppose that $\vartheta(t)$ is the unique solution to the IVP

$$
\begin{aligned}
\vartheta^{\prime \prime \prime} & =f_{3}(t, \vartheta), \\
f_{3}(t, \vartheta) & =q(t) \vartheta(t), \quad \text { with } \quad \vartheta(a)=0, \quad \vartheta^{\prime}(a)=0, \quad \vartheta^{\prime \prime}(a)=1 .
\end{aligned}
$$

Then the linear combination

$$
u(t)=\psi(t)+\theta_{1} \rho(t)+\theta_{2} \vartheta(t),
$$

is a solution to the BVP (1).

For the boundary condition type I, the solution $u(t)$ in equation $(47)$ takes on the boundary values. Then the linear combination

$$
\begin{aligned}
u(a) & =\psi(a)+\theta_{1} \rho(a)+\theta_{2} \vartheta(a), \\
u(a) & =\alpha . \\
u^{\prime}(a) & =\psi^{\prime}(a)+\theta_{1} \rho^{\prime}(a)+\theta_{2} \vartheta^{\prime}(a),
\end{aligned}
$$




$$
\begin{aligned}
u^{\prime}(a) & =\theta_{1} . \\
u(b) & =\psi(b)+\theta_{1} \rho(b)+\theta_{2} \vartheta(b) .
\end{aligned}
$$

Imposing the boundary conditions $u^{\prime}(a)=\beta$ and $u(b)=\gamma$ in (51) and (52) produces $\theta_{1}=\beta$ and $\theta_{2}=\frac{\gamma-\psi(b)-\beta \rho(b)}{\vartheta(b)}$. Therefore, if $\vartheta(b) \neq 0$, the unique solution of the two-point BVP (1) with boundary condition type I is given by:

$$
u(t)=\psi(t)+\beta \rho(t)+\frac{\gamma-\psi(b)-\beta \rho(b)}{\vartheta(b)} \vartheta(t) .
$$

For the boundary condition type II, the solution $u(t)$ in equation (47) takes on the boundary values. Then the linear combination

$$
\begin{aligned}
u(a) & =\psi(a)+\theta_{1} \rho(a)+\theta_{2} \vartheta(a), \\
u(a) & =\alpha, \\
u^{\prime}(a) & =\psi^{\prime}(a)+\theta_{1} \rho^{\prime}(a)+\theta_{2} \vartheta^{\prime}(a), \\
u^{\prime}(a) & =\theta_{1} . \\
u^{\prime}(b) & =\psi^{\prime}(b)+\theta_{1} \rho^{\prime}(b)+\theta_{2} \vartheta^{\prime}(b) .
\end{aligned}
$$

Imposing the boundary conditions $u^{\prime}(a)=\beta$ and $u^{\prime}(b)=\lambda$ in (57) and (58) produces $\theta_{1}=\beta$ and $\theta_{2}=\frac{\gamma-\psi^{\prime}(b)-\beta \rho^{\prime}(b)}{\vartheta^{\prime}(b)}$. Therefore, if $\vartheta^{\prime}(b) \neq 0$, the unique solution of the two-point BVP (1) with boundary condition type II is given by:

$$
u(t)=\psi(t)+\beta \rho(t)+\frac{\gamma-\psi^{\prime}(b)-\beta \rho^{\prime}(b)}{\vartheta^{\prime}(b)} \vartheta(t) .
$$

\section{Algorithm 1: RKT Method via Linear Shooting Technique:}

To approximate the solution of BVP (1) with boundary condition type I:

INPUT: $\alpha, \beta, \gamma$ boundary conditions; $a, b$ endpoints; $N$ number of subintervals. OUTPUT: approximations $\varphi_{1, i}$ to $u\left(t_{i}\right) ; \varphi_{2, i}$ to $u^{\prime}\left(t_{i}\right) ; \varphi_{3, i}$ to $u^{\prime \prime}\left(t_{i}\right)$ for each $i=0,1, \ldots, N$.

Step 1: Set $h=(b-a) / N$;

$$
\begin{aligned}
& \psi_{1,0}=\alpha \\
& \psi_{2,0}=0 ; \\
& \psi_{3,0}=0 ; \\
& \rho_{1,0}=0
\end{aligned}
$$




$$
\begin{aligned}
& \rho_{2,0}=1 ; \\
& \rho_{3,0}=0 ; \\
& \vartheta_{1,0}=0 ; \\
& \vartheta_{2,0}=0 ; \\
& \vartheta_{3,0}=1 .
\end{aligned}
$$

Step 2: For $i=0, \ldots, N-1$ do Step 3 and Step 4 .

(RKD method is used in Step 3 and Step 4.)

Step 3: Set $t=a+i h$.

Step 4: Set

$$
\begin{aligned}
\kappa_{1} & =f_{1}\left(t, \psi_{1, i}\right) ; \\
\kappa_{i} & =f_{1}\left(t+c_{i} h, \psi_{1, i}+c_{i} h \psi_{2, i}+\frac{h^{2}}{2} c_{i}^{2} \psi_{3, i}+h^{3} \sum_{j=1}^{i-1} a_{i j} \kappa_{j}\right) \\
\psi_{1, i+1} & =\psi_{1, i}+h \psi_{2, i}+\frac{h^{2}}{2} \psi_{3, i}+h^{3} \sum_{i=1}^{\mu} b_{i} \kappa_{i} ; \\
\psi_{2, i+1} & =\psi_{2, i}+h \psi_{3, i}+h^{2} \sum_{i=1}^{\mu} \hat{b}_{i} \kappa_{i} ; \\
\psi_{3, i+1} & =\psi_{3, i}+h \sum_{i=1}^{\mu} \hat{\hat{b}}_{i} \kappa_{i} ; \\
\overline{\kappa_{1}} & =f_{2}\left(t, \rho_{1, i}\right) ; \\
\bar{\kappa}_{i} & =f_{2}\left(t+c_{i} h, \rho_{1, i}+c_{i} h \rho_{2, i}+\frac{h^{2}}{2} c_{i}^{2} \rho_{3, i}+h^{3} \sum_{j=1}^{i-1} a_{i j} \bar{\kappa}_{j}\right) ; \\
\rho_{1, i+1} & =\rho_{1, i}+h \rho_{2, i}+\frac{h^{2}}{2} \rho_{3, i}+h^{3} \sum_{i=1}^{\mu} b_{i} \bar{\kappa}_{i} ; \\
\rho_{3, i+1} & =\rho_{3, i}+h \sum_{i=1}^{\mu} \hat{\hat{b}}_{i} \bar{\kappa}_{i} ; \\
\rho_{2, i+1} & \left.=\rho_{2, i}+h \rho_{3, i}+h_{1, i}\right) ; \hat{b}_{i} \bar{\kappa}_{i} ; \\
&
\end{aligned}
$$




$$
\begin{aligned}
\overline{\bar{\kappa}}_{i} & =f_{3}\left(t+c_{i} h, \vartheta_{1, i}+c_{i} h \vartheta_{2, i}+\frac{h^{2}}{2} c_{i}^{2} \vartheta_{3, i}+h^{3} \sum_{j=1}^{i-1} a_{i j} \overline{\bar{\kappa}}_{j}\right) ; \\
\vartheta_{1, i+1} & =\vartheta_{1, i}+h \vartheta_{2, i}+\frac{h^{2}}{2} \vartheta_{3, i}+h^{3} \sum_{i=1}^{\mu} b_{i} \overline{\bar{\kappa}}_{i} ; \\
\vartheta_{2, i+1} & =\vartheta_{2, i}+h \vartheta_{3, i}+h^{2} \sum_{i=1}^{\mu} \hat{b}_{i} \overline{\bar{\kappa}}_{i} ; \\
\vartheta_{3, i+1} & =\vartheta_{3, i}+h \sum_{i=1}^{\mu} \hat{\hat{b}}_{i} \overline{\bar{\kappa}}_{i} ;
\end{aligned}
$$

Step 5: (For boundary condition type I)

$$
\begin{gathered}
\text { set } \varphi_{1,0}=\alpha ; \\
\varphi_{2,0}=\beta ; \\
\varphi_{3,0}=\frac{\left(\gamma-\psi_{1, N}-\varphi_{2,0} \rho_{1, N}\right)}{\vartheta_{1, N}} ; \\
\text { OUTPUT }\left(a, \varphi_{1,0}, \varphi_{2,0}, \varphi_{3,0}\right) .
\end{gathered}
$$

Step 5: (For boundary condition type II)

$$
\begin{gathered}
\text { set } \varphi_{1,0}=\alpha ; \\
\varphi_{2,0}=\beta ; \\
\varphi_{3,0}=\frac{\left(\gamma-\psi_{2, N}-\varphi_{2,0} \rho_{2, N}\right)}{\vartheta_{2, N}} ; \\
\text { OUTPUT }\left(a, \varphi_{1,0}, \varphi_{2,0}, \varphi_{3,0}\right) .
\end{gathered}
$$

Step 6: For $i=1, \ldots, N$ set

$$
\begin{gathered}
\Omega 1=\psi_{1, i}+\varphi_{2,0} \rho_{1, i}+\varphi_{3,0} \vartheta_{1, i} ; \\
\Omega 2=\psi_{2, i}+\varphi_{2,0} \rho_{2, i}+\varphi_{3,0} \vartheta_{2, i} ; \\
\Omega 3=\psi_{3, i}+\varphi_{2,0} \rho_{3, i}+\varphi_{3,0} \vartheta_{3, i} ; \\
\quad t=a+i h ; \\
\text { OUTPUT }(t, \Omega 1, \Omega 2, \Omega 3) .
\end{gathered}
$$

Step 7: Complete. 


\section{Numerical Results}

In this section, we selected four problems to test the performance of the RKT3s4 method in terms of accuracy and effectiveness. The first two problems are BVP problems, while the second problems are a class of BVP called self-adjoint singularly perturbed boundary value problems (SPBVPs).

For the numerical comparisons, we chose the following fourth-order RK types methods of comparisons for the first and second problems, whereas for SPBVPs we wanted to examine whether the new method could solve this type of problem or not, for this we compared with Quartic B-spline methods. Taking into consideration that the Quartic B-spline and Runge-Kutta type methods have different behavior.

All calculations were performed using the code written by us in $\mathrm{C}$ program.

- RKT3s4: The three-stage fourth-order explicit RKT method derived in this paper;

- RKD4M: The three-stage fourth-order RKT method of [23];

- RK4: The four-stage fourth-order RK method given in [22];

- RK4Z: The five-stage fourth-order RK method of [24];

- F.N: The number of function evaluations;

- MAXE: Max $\left(\left|y\left(t_{n}\right)-y_{n}\right|\right)$ which is the maximum between absolute errors of the exact solutions and the computed solutions;

Problem 1. (See Arshad et al. [25]) Consider the inhomogeneous linear twopoint BVP

$$
\begin{aligned}
u^{\prime \prime \prime} & =t u+\left(t^{3}-2 t^{2}-5 t-3\right) e^{t}, \quad 0 \leq t \leq 1, \\
u(0) & =0, \quad u^{\prime}(0)=1, \quad u(1)=0 .
\end{aligned}
$$

The analytic solution is given by

$$
u(t)=t(1-t) e^{t} .
$$

Problem 2. (See Abd El-Salam et al. [26]) Consider the inhomogeneous linear two-point BVP

$$
\begin{aligned}
u^{\prime \prime \prime}+u & =(t-4) \sin t+(1-t) \cos t, \quad 0 \leq t \leq 1, \\
u(0) & =0, \quad u^{\prime}(0)=-1, \quad u^{\prime}(1)=\sin (1) .
\end{aligned}
$$


The analytic solution is given by

$$
u(t)=(t-1) \sin t .
$$

Problem 3. (See Saini et al. [27]) Consider the inhomogeneous two-point SPBVP

$$
\begin{aligned}
-\epsilon u^{\prime \prime \prime}+u & =6 \epsilon(1-t)^{5} t^{3}-6 \epsilon^{2}\left(6(1-t)^{5}-90(1-t)^{4} t\right. \\
& \left.+180(1-t)^{3} t^{2}-60(1-t)^{2} t^{3}\right), \\
u(0) & =0, \quad u^{\prime}(0)=0, \quad u(1)=0 .
\end{aligned}
$$

The analytic solution is given by

$$
u(t)=6 t^{3} \epsilon(1-t)^{5} .
$$

Problem 4. (See Saini et al. [27]) Consider the inhomogeneous two-point SPBVP

$$
\begin{aligned}
-\epsilon u^{\prime \prime \prime}+u & =81 \epsilon^{2} \cos 3 t+3 \epsilon \sin 3 t, \\
u(0) & =0, \quad u^{\prime}(0)=9 \epsilon, \quad u(1)=3 \epsilon \sin (3) .
\end{aligned}
$$

The analytic solution is given by

$$
u(t)=3 \epsilon \sin 3 t .
$$

It is easy to note through Figures 1, 2 that RKT3s4 method performs well when integrating third-order BVP compared to RKD4M, RK4, and RK4Z methods and we can observe from Tables 3, 4 that the numerical results for the RKT3s4 agree to one decimal place when compared with the fourth-order RK methods (RKD4M, RK4, and RK4Z). Add to that, the figures confirm that RKT3s4 method requires fewer function evaluations than RK4 and RK4Z methods. That is because when we solve problems (1), (2) using RK4 and RK4Z methods, we need to reduce them to a system of first-order BVPs which is three times the dimension. Therefore, using a direct method means skipping the step which involves solving the system of linear differential equations which can 


\begin{tabular}{llll}
\hline$N$ & Methods & F.N & MAXE \\
\hline \multirow{4}{*}{4} & RKT3s4 & 12 & $4.290020890(-6)$ \\
& RKD4M & 12 & $2.572186843(-5)$ \\
& RK4 & 48 & $4.653033201(-5)$ \\
& RK4Z & 60 & $4.653033201(-5)$ \\
\hline \multirow{4}{*}{8} & RKT3s4 & 24 & $2.314759256(-7)$ \\
& RKD4M & 24 & $1.595541387(-6)$ \\
& RK4 & 96 & $3.384321949(-6)$ \\
& RK4Z & 120 & $3.384321949(-6)$ \\
\hline \multirow{4}{*}{16} & RKT3s4 & 48 & $8.975345855(-9)$ \\
& RKD4M & 48 & $9.853887617(-8)$ \\
& RK4 & 192 & $2.202264262(-7)$ \\
& RK4Z & 240 & $2.202264262(-7)$ \\
\hline \multirow{3}{*}{32} & RKT3s4 & 96 & $4.998855219(-10)$ \\
& RKD4M & 96 & 5.856636787 \\
& RK4 & 384 & $1.406407724(-8)$ \\
& RK4Z & 480 & $1.406407724(-8)$ \\
\hline
\end{tabular}

Table 3: Maximum errors and number of function evaluations of Problem 1.

save a large amount of work, in terms of the number of function evaluations. As for solving third order SPBVP with respect to different values of $\epsilon$, we observed that the results obtained in Tables 5,8 showed the efficiency of the new method. Figures 3, 4 are also visualized comparing the given tables. Thus, the RKT3s4 method is very efficient and accurate to evaluate according to the given problems and boundary conditions.

\section{Conclusion}

The two-point boundary value problems of third-order ordinary differential equations with boundary conditions type I and type II, can be solved using the direct method of Runge-Kutta via shooting technique. The experiments have shown that the new method worked well, as expected, which is to say that using a direct method to solve the higher-order ordinary differential equations is not only most efficient in terms of the absolute maximum global error but is 


\begin{tabular}{lllll}
\hline$N$ & Methods & F.N & MAXE \\
\hline \multirow{4}{*}{4} & RKT3s4 & 12 & $1.967463006(-6)$ \\
& RKD4M & 12 & $1.799932279(-5)$ \\
& RK4 & 48 & $1.159839228(-4)$ \\
& RK4Z & 60 & $1.159839228(-4)$ \\
\hline \multirow{4}{*}{8} & RKT3s4 & 24 & $1.869702460(-7)$ \\
& RKD4M & 24 & $1.549408651(-6)$ \\
& RK4 & 96 & $7.166950172(-6)$ \\
& RK4Z & 120 & $7.166950172(-6)$ \\
\hline \multirow{4}{*}{16} & RKT3s4 & 48 & $5.482110596(-8)$ \\
& RKD4M & 48 & $1.543556326(-7)$ \\
& RK4 & 192 & $4.432972907(-7)$ \\
& RK4Z & 240 & $4.432972907(-7)$ \\
\hline \multirow{3}{*}{32} & RKT3s4 & 96 & $8.907090702(-9)$ \\
& RKD4M & 96 & $1.693722997(-8)$ \\
& RK4 & 384 & $2.752917824(-8)$ \\
& RK4Z & 480 & $2.752917824(-8)$ \\
\hline \multirow{4}{*}{} & & &
\end{tabular}

Table 4: Maximum errors and number of function evaluations of Problem 2.

\begin{tabular}{cccc}
\hline$N$ & $\epsilon=1 / 16$ & $\epsilon=1 / 32$ & $\epsilon=1 / 64$ \\
\hline 10 & $6.5 \times 10^{-6}$ & $2.8 \times 10^{-6}$ & $1.0 \times 10^{-6}$ \\
20 & $4.2 \times 10^{-7}$ & $1.7 \times 10^{-7}$ & $6.6 \times 10^{-8}$ \\
40 & $2.6 \times 10^{-8}$ & $1.1 \times 10^{-8}$ & $4.1 \times 10^{-9}$ \\
\hline
\end{tabular}

Table 5: Maximum error of RKT3s4 of Problem 3.

more efficient as it can save a large amount of work, in terms of the number of function evaluations. 


\begin{tabular}{cccc}
\hline$N$ & $\epsilon=1 / 16$ & $\epsilon=1 / 32$ & $\epsilon=1 / 64$ \\
\hline 10 & $4.7 \times 10^{-4}$ & $1.9 \times 10^{-4}$ & $8.0 \times 10^{-5}$ \\
20 & $1.1 \times 10^{-4}$ & $4.7 \times 10^{-5}$ & $1.9 \times 10^{-5}$ \\
40 & $2.6 \times 10^{-5}$ & $1.2 \times 10^{-5}$ & $4.8 \times 10^{-6}$ \\
\hline
\end{tabular}

Table 6: Maximum error of Problem 3 in Saini [27].

\begin{tabular}{cccc}
\hline$N$ & $\epsilon=1 / 16$ & $\epsilon=1 / 32$ & $\epsilon=1 / 64$ \\
\hline 10 & $2.9 \times 10^{-3}$ & $9.2 \times 10^{-4}$ & $1.4 \times 10^{-4}$ \\
20 & $1.2 \times 10^{-4}$ & $3.8 \times 10^{-5}$ & $6.8 \times 10^{-6}$ \\
40 & $6.4 \times 10^{-6}$ & $2.1 \times 10^{-6}$ & $4.6 \times 10^{-7}$ \\
\hline
\end{tabular}

Table 7: Maximum error of Problem 3 in Akram [28].

\begin{tabular}{cccc}
\hline$N$ & $\epsilon=1 / 16$ & $\epsilon=1 / 32$ & $\epsilon=1 / 64$ \\
\hline 10 & $1.9 \times 10^{-5}$ & $3.7 \times 10^{-5}$ & $7.0 \times 10^{-5}$ \\
20 & $2.6 \times 10^{-6}$ & $5.0 \times 10^{-6}$ & $9.9 \times 10^{-6}$ \\
40 & $3.4 \times 10^{-7}$ & $6.6 \times 10^{-7}$ & $1.3 \times 10^{-6}$ \\
\hline
\end{tabular}

Table 8: Maximum error of RKT3s4 of Problem 4.

\begin{tabular}{cccc}
\hline$N$ & $\epsilon=1 / 16$ & $\epsilon=1 / 32$ & $\epsilon=1 / 64$ \\
\hline 10 & $2.4 \times 10^{-4}$ & $1.0 \times 10^{-4}$ & $4.0 \times 10^{-5}$ \\
20 & $6.1 \times 10^{-5}$ & $2.6 \times 10^{-5}$ & $1.0 \times 10^{-6}$ \\
40 & $1.5 \times 10^{-5}$ & $6.4 \times 10^{-6}$ & $2.5 \times 10^{-6}$ \\
\hline
\end{tabular}

Table 9: Maximum error of Problem 4 in Saini [27].

\section{Acknowledgements}

This study has been supported by the Fundamental Research Grant Scheme (Ref. No. FRGS/1/2018/STG06/UPM/02/2) awarded by the Malaysia Min- 


\begin{tabular}{cccc}
\hline$N$ & $\epsilon=1 / 16$ & $\epsilon=1 / 32$ & $\epsilon=1 / 64$ \\
\hline 10 & $1.3 \times 10^{-2}$ & $3.2 \times 10^{-3}$ & $3.4 \times 10^{-4}$ \\
20 & $1.1 \times 10^{-3}$ & $2.7 \times 10^{-4}$ & $2.2 \times 10^{-5}$ \\
40 & $7.8 \times 10^{-5}$ & $1.8 \times 10^{-5}$ & $1.1 \times 10^{-6}$ \\
\hline
\end{tabular}

Table 10: Maximum error of Problem 4 in Akram [28].

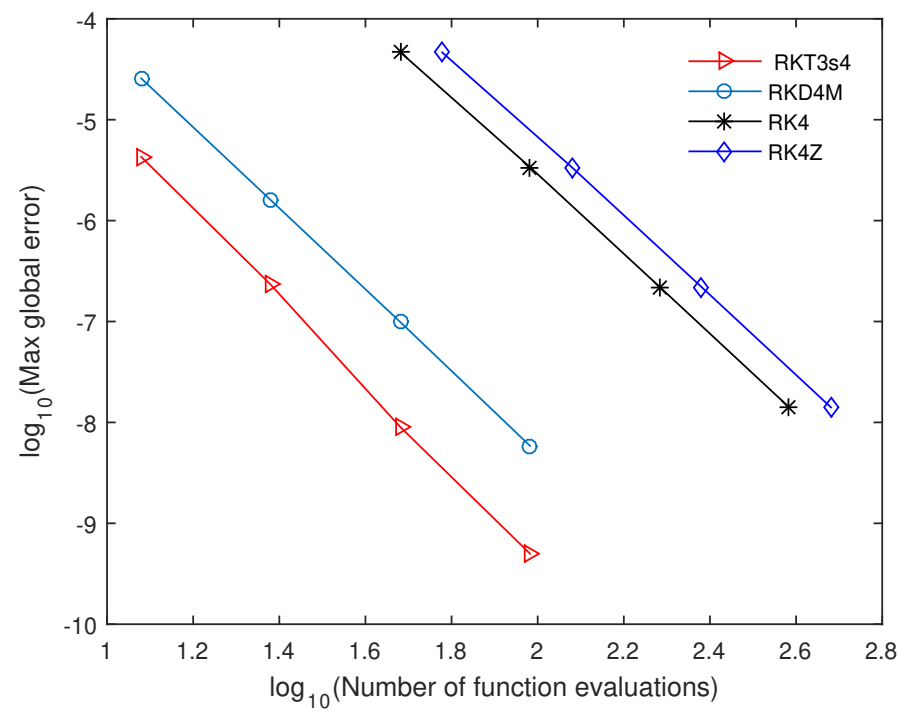

Figure 1: The efficiency curve for RKT3s4, RKD4M, RK4, and RK4Z for Problem 1 with $N=2^{i}, i=2,3,4,5$.

istry of Education.

\section{References}

[1] R.L. Burden, J.D. Faires, Numerical Analysis, Brooks/Cole, Cengage Learning, Boston (2011).

[2] T.S. Mohamed, N. Senu, Z.B. Ibrahim and N.M.A. Nik Long, Efficient two-derivative Runge-Kutta-Nyström methods for solving general second- 


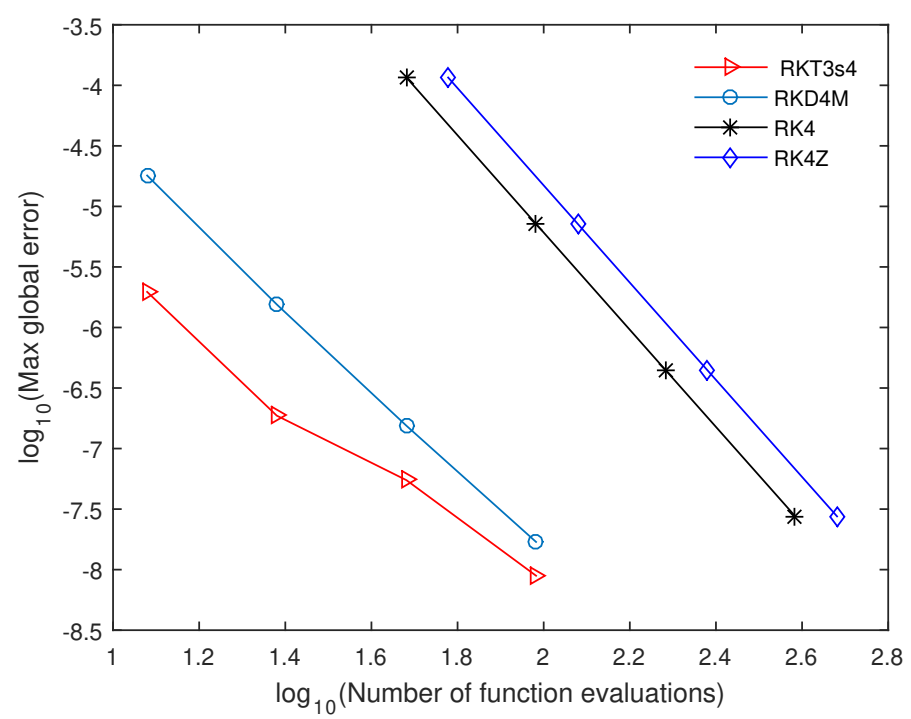

Figure 2: The efficiency curve for RKT3s4, RKD4M, RK4, and RK4Z for Problem 2 with $N=2^{i}, i=2,3,4,5$.

order ordinary differential equations, Discrete Dynamics in Nature and Society, 2018 (2018), 10 pages.

[3] N. Ghawadri, N. Senu, F. Ismail and Z.B. Ibrahim, Exponentially fitted and trigonometrically fitted explicit modified Runge-Kutta type methods for solving $y^{\prime \prime \prime}=f\left(x, y, y^{\prime}\right)$, J. of Applied Mathematics, 2018 (2018), 19 pages.

[4] T.Y. Na, Computational Methods in Engineering Boundary Value Problems, Academic Press, New York (1980).

[5] U.M. Ascher, R.M.M. Mattheij and R.D. Russell, Numerical Solution of Boundary Value Problems for Ordinary Differential Equations, SIAM, Philadelphia (1994).

[6] P. Henrici, Discrete Variable Methods in Ordinary Differential Equations, Wiley, New York (1962).

[7] C. Kanittha and A. Dhamacharoen, Solving boundary value problems of ordinary differential equations with non-separated boundary conditions, Applied Mathematics and Computation, 217, No 24 (2011), 10355-10360. 


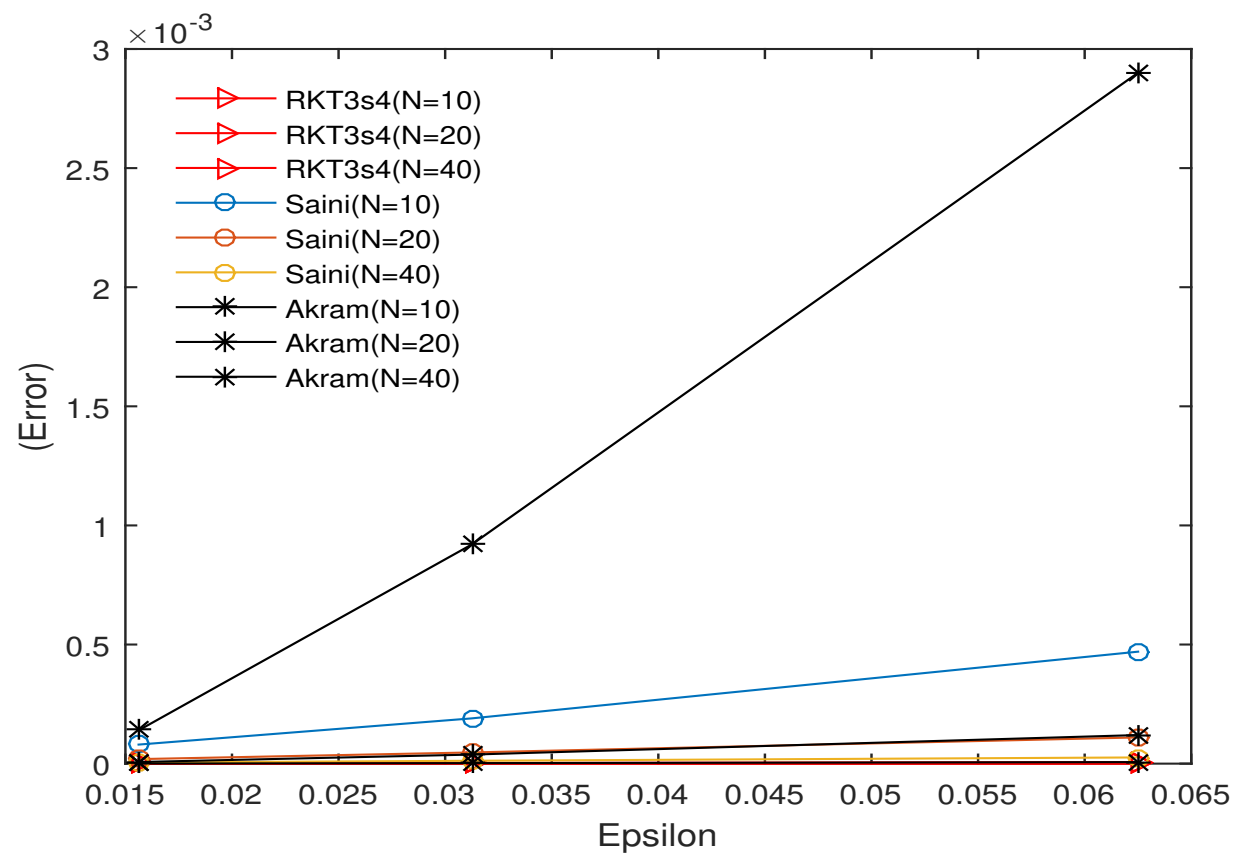

Figure 3: Comparison figure between RKT3s4, Saini, and Akram methods.

[8] A. Dhamacharoen and K. Chompuvised, An efficient method for solving multipoint equation boundary value problems, World Academy of Science, Engineering and Technology, 75 (2013), 61-65.

[9] I.A. Tirmizi and E.H. Twizell, Higher-order finite-difference methods for nonlinear second-order two-point boundary-value problems, Applied Mathematics Letters, 15, No 7 (2002), 897-902.

[10] A. Pierluigi and I. Sgura, High-order finite difference schemes for the solution of second-order BVPs, J. of Computational and Applied Mathematics, 176, No 1 (2005), 59-76.

[11] S.N. Ha, A nonlinear shooting method for two-point boundary value problems, Computers and Mathematics with Applications, 42, No 10 (2001), 1411-1420.

[12] B. Ahmad, J.J. Nieto and N. Shahzad, Generalized quasilinearization 


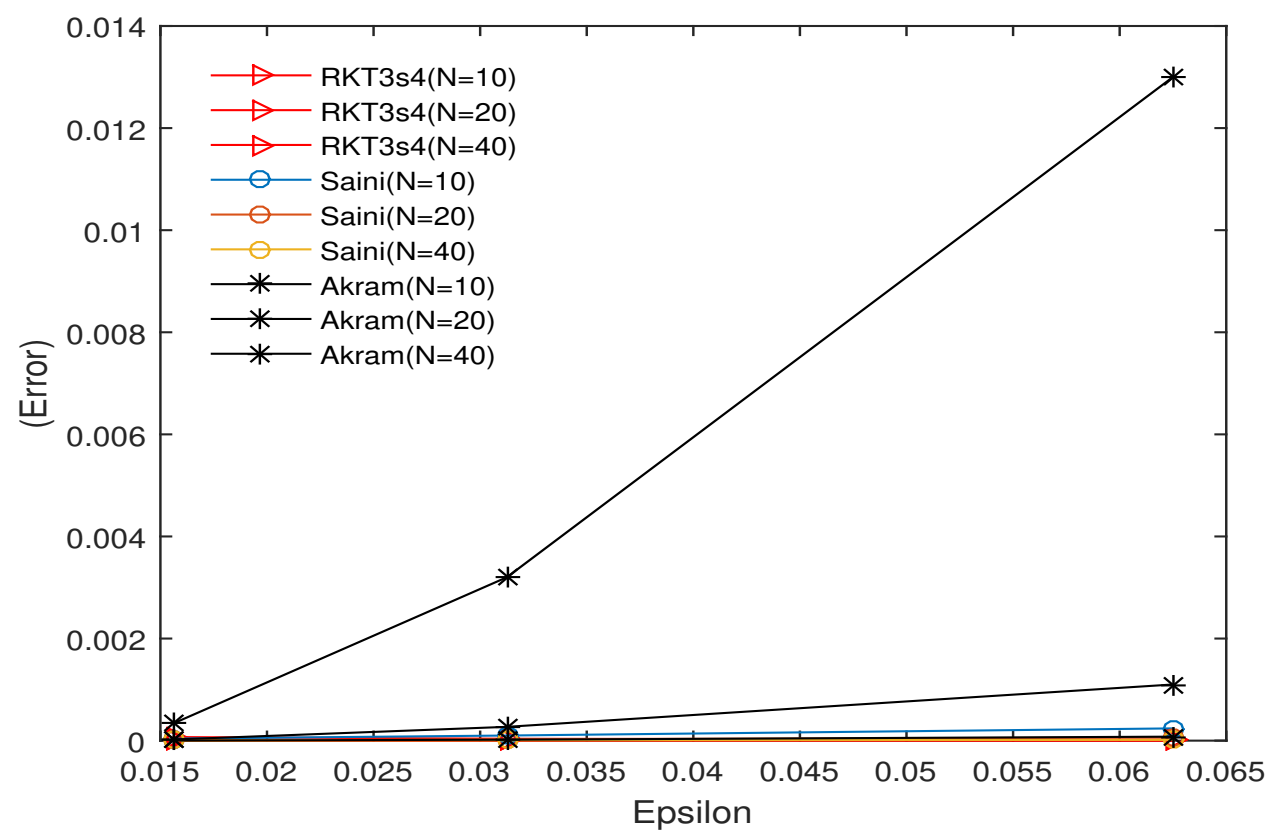

Figure 4: Comparison figure between RKT3s4, Saini, and Akram methods.

method for mixed boundary value problems, Applied Mathematics and Computation, 133, No 2 (2002), 423-429.

[13] R.A. Khan, The generalized quasilinearization technique for a second order differential equation with separated boundary conditions, Mathematical and Computer Modelling, 43, No 7 (2006), 727-742.

[14] M. Cherpion, C. De Coster and P. Habets, A constructive monotone iterative method for second-order BVP in the presence of lower and upper solutions, Applied Mathematics and Computation, 123, No 1 (2001), 75-91.

[15] P.W. Eloe and Y. Zhang, A quadratic monotone iteration scheme for twopoint boundary value problems for ordinary differential equations, Nonlinear Analysis: Theory, Methods and Applications, 33, No 5 (1998), 443-453.

[16] J. Lu, Variational iteration method for solving two-point boundary value problems, J. of Computational and Applied Mathematics, 207, No 1 (2007), 92-95. 
[17] Z.M. Odibat and S. Momani, Variational iteration method for solving nonlinear boundary value problems, Applied Mathematics and Computation, 183, No 2 (2006), 1351-1358.

[18] S.N. Jator, Numerical integrators for fourth order initial and boundary value problems, International J. of Pure and Applied Mathematics, 47, No 4 (2008), 563-576.

[19] K. Hussain, F. Ismail and N. Senu, Solving directly special fourth-order ordinary differential equations using Runge-Kutta type method, J. of Computational and Applied Mathematics, 306 (2016), 179-199.

[20] M. Mechee, N. Senu, F. Ismail and B. Nikouravan, A three-stage fifthorder Runge-Kutta method for directly solving special third-order differential equation with application to thin film flow problem, Mathematical Problems in Engineering, 2013 (2013), 7 pages.

[21] J.R. Dormand and P.J. Prince, A family of embedded Runge-Kutta formulae, J. of Computational and Applied Mathematics, 6, No 1 (1980), 19-26.

[22] J.C. Butcher, Numerical Methods for Ordinary Differential Equations, John Wiley and Sons Ltd., England (2008).

[23] M. Mechee, F. Ismail, Z.M. Hussain and Z. Siri, Direct numerical methods for solving a class of third-order partial differential equations, Applied Mathematics and Computation, 247 (2014), 663-674.

[24] E. Hairer, S.P. Nrsett and G. Wanner, Solving Ordinary Differential Equations, Springer-Verlag, Berlin (1993).

[25] A. Khan and T. Aziz, The numerical solution of third-order boundaryvalue problems using quintic splines, Applied Mathematics and Computation, 137, No 2 (2003), 253-260.

[26] F.A.A. El-Salam, A.A. El-Sabbagh and Z.A. Zaki, The numerical solution of linear third order boundary value problems using nonpolynomial spline technique, J. of American Science, 6, No 12 (2010), 303-309.

[27] S. Saini and H.K. Mishra, A new quartic B-spline method for third order self-adjoint singularly perturbed boundary value problems, Applied Mathematical Sciences, 9, No 8 (2015), 399-408. 
[28] G. Akram, Quartic spline solution of a third order singularly perturbed boundary value problem, ANZIAM J., 53 (2012), 44-58. 